\title{
Increasing Student Engagement with Personalized Emails
}

\author{
Bshaer Alwagdani ${ }^{1} \&$ Khalid Alomar ${ }^{1}$ \\ ${ }^{1}$ Department of Information Systems, King Abdulaziz University, Jeddah, Saudi Arabia \\ Correspondence: Bshaer Alwagdani, Department of Information Systems, King Abdulaziz University, Jeddah, \\ Saudi Arabia; Khalid Alomar, Department of Information Systems, King Abdulaziz University, Jeddah, Saudi \\ Arabia.
}

\author{
Received: March 30, 2020 \\ Accepted: April 13, $2020 \quad$ Online Published: April 29, 2020 \\ doi:10.5539/cis.v13n2p54 \\ URL: https://doi.org/10.5539/cis.v13n2p54
}

\begin{abstract}
Despite a large number of studies in personalizing e-learning systems, only a few sufficiently cover the impact of personalization on university emails. Either universities impose the use of university email or students are reluctant to use it. For instance, It was found that at King Abdulaziz University only 23\% of students were using the university email to communicate regularly, unlike the faculty members, who showed an $80 \%$ commitment. However, this paper attempts to investigate the effect of applying different personalized systems on the university email and whether using adaptation and adaptability techniques in the university email will cover the huge gap between the university and its members. Specifically, frameworks were built to evaluate the efficiency, frequency of error occurrence, effectiveness, and user satisfaction in each experiment. We focused on testing the usability of personalized email against the existing university email and then evaluate which approach (adaptive, adaptable, or mixed-initiative) is more favorable to personalize the university email. These were conducted and evaluated using 40 subjects. Interestingly, results show that subjects with personalized emails were most efficient and satisfied as well as errors were reduced by $42 \%$. Furthermore, significant differences were found between the three approaches (adaptive, adaptable, and mixed-initiative), and the adaptive approach was the most preferred by the respondents. A set of empirically derived guidelines was also discussed as a basis for developing a suitable university email structure.
\end{abstract}

Keywords: personalization, adaptive, adaptable, mixed-initiative

\section{Introduction}

The success of a web service greatly depends on its interface quality(Anabalon, Flores, Mateos, Zunino, \& Misra 2017) . Furthermore, the proliferation of user interfaces and functional complexity can affect user efficiency, precision, and mood (Wu, Yu, Wei, Xie, \& Kishida, 2010). So improving the quality of user interface is crucial to meet users' expectations. E-learning systems use different personalization technologies to overcome the gap between learners and educational systems. Accordingly, it has to be added for better achievements (Jumaa, Moussa, \& Khalifa, 2017) . Several types of adaptations (Soflano, Connolly, \& Hainey, 2015) (Connolly \& Stansfield, 2006) (Sabourin \& Lester, 2014) (Federico, 2000) (Graf \& Kinshuk, 2007) (Nigel \& Chen, 2000) (Burgos, Tattersall, \& Koper, 2007) (Akbulut \& Cardak, 2012) are considered as essential techniques in personalized systems: adaptive, which change the interface content and layout; adaptable, which allow users to adjust the interface according to their need; and mixed approach, which combine these two conditions. Other study (Srivastava \& Goyal, 2013) claimed that many available university systems are complex and fail to preserve a high standard in terms of their interface and functionalities. A large volume of websites and web applications are available for different users. But Defining which requirements are fundamental to a particular system (communication, shopping, etc.), in addition to several usability problems, are the key factors (Kaur \& Sharma, 2018). Nevertheless, even if many developers persevere to make an appropriate approach with applicable services, the user is the one who has the last opinion regarding what services are suitable to his need (Mateos, Zunino, Misra, Anabalon, \& Flores, 2016).

The primary contribution of this study is to maintain an experimental evaluation regarding the impact of different personalized technologies on the university e-mail system. Firstly, the study evaluates the system's efficiency and the effectiveness of personalized e-mail versus the existing university e-mail system and then, at the second stage, measures the effectiveness, efficiency, and overall user satisfaction of (adaptable, adaptive, and mixed approach) to determine which approach is best and suits the need of King Abdul-Aziz University e-mail users. 


\subsection{Personalization}

First Personalization is the process of adapting a system's content, interface, and functionality to suit user need (Findlater \& McGrenere, 2010). It can be accomplished through two approaches, the adaptable and the adaptive approach, which differ in terms of who is responsible for controlling the customization. The adaptable approach grants the user ability to adjust the system's layout and content through customization techniques, while under the adaptive approach, the system adapts its behavior (content and interface) depending on what the user needs (McDonald, Molander, \& Noel, 1988). Questions have been raised about which approach is the better way to personalize (Shneiderman \& Maes, 1997). Each approach has its own benefits and weaknesses. Not all users want full customization control, and not all users want to rely entirely on the system to control the interface. For this reason, some researchers have suggested integrating both techniques into a mixed-initiative approach, so as to modify their weaknesses and make the most of their advantages (Cecchinato, Bird, \& Cox, 2000) (Findlater \& McGrenere, 2004).

However, various educational institutions emphasize the importance of developing new types of learning technologies to meet students' needs (McLoughlin \& Lee, 2008). It is claimed that if the administrators apply useful personalized technology in education and communication systems, students will be more engaged with the online learning environment (Betts, 2009).

Research on personalising system behaviour to user interactions involves many fields, such as adaptive hypermedia and hypertext, web personalisation, information filtering, recommender systems, and personalised information retrieval (Zanker, Rook, \& Jannach, 2019). The aspects of personalising content include the following: adding, editing, deleting, and categorizing email content, in addition to customising news and announcements. Furthermore, personalizing a GUI involves the following: changing the appearance of message types in a mailbox (such as section messages), the personal signature, folders, and themes. The dimensions of design space as concerns personalization and adaptation mechanisms consists of the following aspects:

\subsubsection{Personalized Graphical User Interface}

A user interface is composed of one or more layers, including physical input components and adaptation control functionality, in order to adapt to the interactions between users and systems (Zanker, Rook, \& Jannach, 2019). Adapting the user interface (UI) promises to enhance user experience (UX) in a massive range (Lim, Hooshyar, $\mathrm{Ji}$, Lee, \& Jo, 2018). When applying user interface adaptations, one must consider many physical aspects, such as movements and geolocation. There are different ways to personalize a graphical user interface (Bunt, 2007), for instance, by applying features such as a menu, a toolbar, and icons to the interface, or through macro mechanisms to produce sequences of functions (MacLean, Carter, Lövstrand , \& Moran, 1990) (Page, Johnsgard, Albert, \& Allen, 1996).

\subsubsection{Personalized Content}

Content adaptation depends on deciding what content is most suitable and related to each user before adapting it (Demchuk, Lytvyn, Vysotska, \& Dilai, 2019). It includes many forms of objects such as news, images, articles, resources, products, media, or fine-grained differences in textual wordings. On the other hand, adaptations in the content displayed are decided based on how to present the exact content to certain users (Bunt, Carenini, \& Conati, 2007). Personalizing content has been successful in many areas, for instance, intelligent tutoring systems, which adapt educational material to suit users' preferences (Chabay \& larkin, 2020) (Almasri et al., 2019); recommender systems (Murali, Vishnu, \& Victor, 2019); or personalizing products in e-commerce systems (Zhao, 2019).

\subsection{Personalized Approaches}

Personalization can be seen as creating a base for information that can be easily reached at any time and from any place. Furthermore, the personalized system removes all the constraints of time and place from the learning experience. Besides its potential to optimize students' abilities in a productive and meaningful way, it can integrate differences between students in e-learning systems (Mateos, Zunino, Misra, Anabalon, \& Flores, 2016).

\subsubsection{Adaptive Approach}

An adaptive system changes its behavior in response to the user's actions. Greenberg and Witten (Gajos, Czerwinski, Tan, \& Weld, 2006), presented the first successful study of adaptation in 1985, when they revealed an adaptive interface for a menu-driven application. Nowadays, there are many examples of adaptive systems (Greenberg, \& Witten, 1985). For instance, in Windows XP, the Start menu provides a small number of shortcuts, and produces extra shortcuts to the most commonly used programs. Another example is MS Word 2000, which introduced a smart toolbar that can display a small list of the most frequently used items. Then, after a certain 
amount of time, it will display the full version of all programs. Microsoft Office proposed another example where recently used fonts are found above a line in the font selection menu and the rest are located below the line in alphabetical order (Sears \& Shneiderman, 1998). A final example of an adaptive approach is found in Amazon.com, which recommends products based on a user's purchase history. It tracks and monitors a user's clicks to provide them with products they are likely to be interested in (Wu, Aggarwal, \& Yu, 2000).

Many researchers have attempted to experimentally evaluate adaptive interface systems. For example, two adaptation techniques were examined with the list of textual selections (Tsandilas, 2005). The first technique highlights the background of suggested items in a variant color, and the other technique minimizes the non-suggested items while letting the users explore the suggested ones by means of a virtual fisheye lens. The results revealed that accuracy affected both user performance and user ability to detect specific items the system suggested. Another study experimentally examined the impact of accuracy and predictability on adaptive interface usability. The results showed that those factors (predictability and accuracy) help to increase satisfaction and performance. In a more precise experiment, three adaptive graphical interface techniques were examined against a non-adaptive baseline (Paterno \& Mancini, 1999). The authors evaluated the three techniques (visual pop-out, moving, and split) using 26 participants, and compared the results with those of other relevant studies (Page, Johnsgard, Albert \& Allen, 1996) (Sears \& Shneiderman, 1998) (Greenberg, \& Witten, 1985). Moreover, they proposed a number of important factors that affect the performance of any adaptive interface system, which can be defined by the frequency of adaptation, accuracy, spatial stability, the frequency of interaction with the interface, and the complexity of the tasks and of the interface itself.

\subsubsection{Adaptable Approach}

A number of studies have been conducted on many adaptable systems. Page et al. studied the type and amount of customization that users take advantage of. They found that $92 \%$ of members customized their (WordPerfect 6.0) software in minor ways, such as displaying or hiding a ruler bar or making shortcuts, over a 28-day period. Nevertheless, the results revealed that customization had become easy enough to be used constantly. In particular, the study showed that $16 \%$ of subjects made macros and 55\% created shortcuts (Findlater \& McGrenere, 2004).

Two studies by Mackay examine user aims and other factors that affect customization (Mackay, 1991). The first study had 51 participants and the second had 18. The first found that users may avoid customizing systems for reasons such as being busy and lacking enough information. And there are also encouraging factors, such as software upgrades, social pressure, and certain external and internal factors (e.g. job changes, extra free time). Moreover, this study revealed that more than $78 \%$ of participants made a certain type of customization.

In another investigation, Mackay (Mackay, 1990). tried to distinguish users who customized a system from those who did not. The users were divided into two groups. The first group was made up of highly skilled software engineers. The second group was made up of translators, considered less skilled than the engineers. The participants were situated on diverse research sites and worked with two different pieces of customizable software. The highly skilled group did not try to make customizations according to the second group's needs, while the second group did try to make customizations according to the other group's needs.

Another study (MacLean, Carter, Lövstrand, \& Moran, 1990) argued that the skill-level of the users could affect the customizations they make. The users were classified into three categories (programmers, thinkers, and workers). The study found that programmers did expect to customize a system. Workers, however, did not expect to customize a system. Thinkers fell in between the two groups.

Several studies investigating adaptability have promoted the idea of multilayer interfaces, where the users can change the interface (McGrenere, 2002) (Shneiderman, 2003). This technique allows beginning users to start at the first layer and move to a higher one when required by expanding the customization capabilities. Each layered interface has been seriously evaluated and used; multilayer interfaces require more careful design to meet the user's needs. One example was a study by Findlater and McGrenere that distinguished between multilayered approaches and a controlled interface. It showed that users prefer layered interfaces over full interfaces alone in terms of findability, while users of multilayered interfaces are more aware of the advanced features in the controlled interface. Furthermore, the study showed the ability of layered interfaces to raise users' performance as compared to full interfaces (Findlater \& McGrenere, 2007).

\subsubsection{Mixed-initiative Approach}

The Many systems today adopt a mixed approach to adaptability. One example of this is the Flex Excel system, which mixes adaptivity and adaptability by providing suggestions to users. It suggests new shortcuts and menu entries for regularly used items and helps the user by providing information on customizing critical features. 
These techniques depend on the rule-based frequency approach. If there are any new suggestions, the user will be notified by a sound and blinking icon that contains a tip up to three times. Thirteen participants examined the system, and some of them met with difficulties in initiating the customizations (Cypher, 1991).

Another example of such a system is a multilayer user interface that assists a user by providing suggestions on choosing either a command-line interface or menu-based interface (Clark \& Matthews, 2005). The recommendations depend on the user's computer experience and the number of user errors. Furthermore, users have the ability to choose the layer they desire to use. But the system, at the same time, provides some visible layers based on the user's editing history. Nevertheless, the study did not provide any evaluation on its effectiveness.

A mixed-initiative approach can be the solution for complexity issues; it can support interface customization to reduce graphical user interface complexity (Bunt, Conati, \& McGrenere, 2007). The researchers examined two interfaces: a typical MS Word interface and a feature-reduced version of the same interface. This contained only some features which the user had to choose to add. The results revealed that the system's recommendations were helpful in improving task performance, and the mixed-initiative approach was more favored than a purely adaptable approach.

\section{Impirical Comparison of Personalized Approaches}

After Preliminary work on different menu conditions was undertaken by (Findlater \& McGrenere, 2007), who compared three different menu conditions - adaptive, adaptable, and static - in terms of their efficiency. Each condition was implemented as a split menu, keeping the different items static and allowing frequent adaptivity and adaptability by the users and their recently used items. The study used 27 respondents to measure the system's performance. Surprisingly, the results showed that users were satisfied with adaptable menus; furthermore, static menus were found to be faster than adaptive menus, and $55 \%$ of subjects favoured the adaptable menus, followed by adaptive menus with $30 \%$ and static with only $15 \%$. With the rapid progress in educational technologies, customization and adaptivity have become essential topics in E-learning systems. Eiman and Meziane (Aeiad, \& Meziane, 2018), described the advances in providing an adaptable learning system that outputs the exact material that suits the learner. They developed the APELS application to extract from the web the right educational materials by using a learners model (background, needs, and learning path). The content that was extracted was evaluated by natural language processing to produce a suitable subject. The system was tested by a domain expert based on the Association for Computing Machinery and IEEE. As a result, the final content was described as high-quality and satisfied the users in the learning environment. In another major study, another study by (Al-Shumari \& Bella, 2014), proposed a vocabulary-learning website for nonEnglish speakers to compare the difficulties for the user of learning vocabulary by using adaptive, adaptable, mixed-initiatives, and static approaches, in addition to the usability of the proposed system to manage learning methods. The tasks were divided into complex, easy, and medium levels. Then, they tested the usability by measuring the efficiency and effectiveness with 24 respondents, while exploring satisfaction with 99 respondents. The presented results were positive. A study by (Jorritsma, Cnossen, \& van Ooijen, 2015), questioned the usefulness of an adaptive approach by proposing adaptive suggestions to evaluate the efficiency of an adaptable Picture Archiving and Communication System (PACS) in a natural work environment. The customization was created based on behavioural user data to support the PACS, which could be ignored or accepted by the respondents (radiologists). Twelve respondents executed the adaptable version of PACS, and only half of them recorded their actions to generate customization support based on their activities. Furthermore, all responses and performances were measured. The findings were interesting and positive regarding the usefulness of PACS with the adaptive support technique. (Frias-Martinez, Chen, \& Liu, 2009) investigated users' interactions with adaptable and adaptive approaches on a standard digital library system. They developed a personalized DL that included two styles: adaptive and adaptable approaches that suited the requirements of different cognitive styles. The results indicate that users performed better with an adaptive approach than with the adaptable version. Furthermore, they had a positive views on the proposed system. In the subsequent study, (Leiva-Chinchilla, Vega-Alvarado, Calvo-Elizondo, \& Francesa-Alfaro, 2018) proposed an adaptative learning prototype in order to facilitate better navigation for LMS users. The system obtained their information such as user model, calendar, evaluation module, and navigation history to adapt the UI depending on each user's requirements. Furthermore, the adaptative system was tested by 20 users via a usability test, and the results were very positive and useful for the developed approach. In another study, (Al-Omar \& Rigas, 2009) built and compared the usability of five interactive menu approaches: adaptive, adaptable split, adaptive/adaptable highlighted, mixed approach, and adaptable/adaptive minimized systems. The study evaluated the efficiency regarding frequency of errors and task accomplishment time by testing 30 respondents. The final results were interesting and proved the usefulness of 
adaptable menus in terms of their high efficiency and few average errors, in contrast to adaptive minimized systems and the mixed condition, which recorded low performance. $\mathrm{Ji}$ et al., (Ji, Yun, Lee, Kim, \& Lim, 2017) were interested in adapting a user interface based on continuous accurate user analysis. They proposed a unique method to analyse a user's behaviour in a distributed environment, then modify the user's profile to provide a customized UI and user experience. In order to support the analysis, they used a cognitive response measurement and 122 user models. Then, the proposed prototype was evaluated by 200 subjects. The overall results were useful and satisfactory.

\section{Experimental Hypothesis}

The purpose of the study is to compare the usability of adaptive, adaptable, mix and static personalized email versus current university email in King Abdulaziz University. With regard to (efficiency, effectiveness, the frequency of error occurrence and user satisfaction), from the previous literature review, the following hypotheses were developed, to be tested by the study. The hypotheses are:

H1: The performance of personalized email will be more efficient than university email regard of task criterion time, frequency of errors, total number of users and tasks completed successfully and user satisfaction.

$\mathrm{H} 2$ : The performance of adaptive condition will be more efficient than both adaptable and mix condition regard of task criterion time, frequency of errors, total number of users and tasks completed successfully and user satisfaction.

H3: The performance of mix condition approaches will be more efficient than both adaptable and adaptive condition regard of task criterion time, frequency of errors, total number of users and tasks completed successfully and user satisfaction.

H4: The performance of adaptable condition approaches will be more efficient than both mix and adaptive. condition regard of task criterion time, frequency of errors, total number of users and tasks completed successfully and user satisfaction.

\section{Method}

This research focused on testing the usability of static personalized university email by King Abdulaziz University users. The general population consisted of 40 subjects (females) from the Faculty of Computing and Information Technology, who were students between the ages of 18 and 35 who used the university email for 4 hours or more each week. We assumed that these subjects had never customized or used the personalized university email. All subjects are divided into four separate groups of ten subjects each concerns different condition. The first ten were test static personalized email condition versus usual university email, in another hand the rest three groups were test three personalized conditions (adaptive, adaptable, mix). The reason for choosing this number of samples was that we found it would provide us with clear indications of all the advantages and any weaknesses of each approach in the initial comparison. All subjects in each system were asked to accomplish the same collection of tasks (easy, medium, difficult), in addition to one learnable task that began before starting any level of tasks. Each user initiated a three-minute training session before performing the required tasks.

Apparatus: In both experiments one and tow a desktop applications was developed using ASP.NET in personal computer.

\subsection{Experimental Design}

All respondents were required to complete the same set of tasks and a single learnable task. Respondents were informed that they had a learnable task before beginning each group; the goal of the learnable task was to support all respondents' ability to accomplish the requested tasks. If the tasks were designed with the same order and complexity for each subject, we couldn't avoid the consequence of the learning effect; thus, the main tasks were created at three levels (easy, medium, and complex), and their arrangement was diverse between subjects. Additionally, to ensure diversity of complexity, we changed some aspects of task scenarios for each group, such as the course name, professor's name, or the type of folder's name. Furthermore, this diversity relied on a number of guidelines when designing the experiments.

In experiment 1 , all the same tasks were executed in both the university and the personalized email. The easy levels consisted of three tasks: subjects would search their mailbox for the last announcement from the university and another from a section; choose the news and announcement depending on certain category; and change the interface to a new theme. In the case of medium tasks, the subject would make her own signature and include her information (college, department, student ID, name, gender), as well as sort all incoming emails automatically 
into different folders based on (i.e. university/college). Finally, for the difficult tasks, the subject would send an email message to two of her professors and type "hello", send an email message to one of her colleagues, and create her own signature. Figure 1 and 2, shows the difference between personalized and university email user interface.

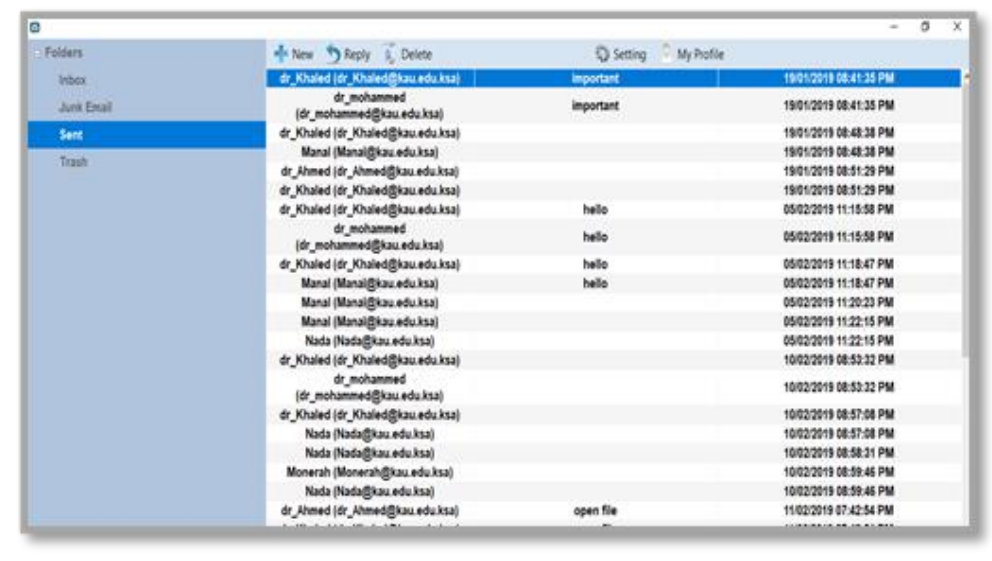

Figure 1. Main user interface in personalized email

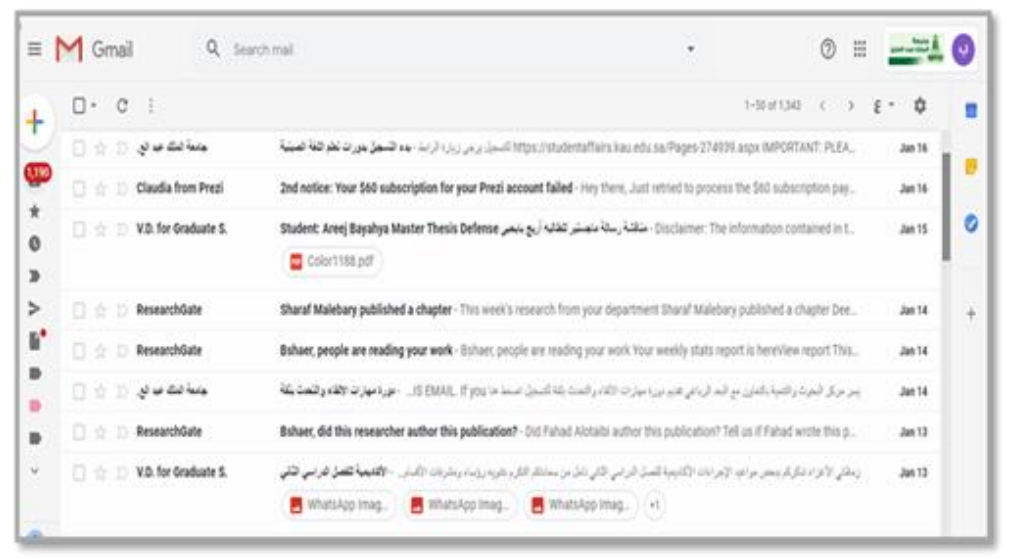

Figure 2. Main user interface in university email

For experiment 2, at the easy level, subjects executed two tasks: changing the interface to a new theme and choosing the announcements and news that they would receive in their email by certain category. Moreover, at the medium level, subjects sorted all emails automatically into different folders ( see figure 3). At the last complexity level, subjects executed four different tasks: send an email message to tow doctors to say hello, and make sure to add a signature; send an email message to one doctor and one of student colleagues to say hello, and make sure to add a signature; send an email message to one of student colleagues in certain course, add an attachment, and make sure to add a signature; and send an email message to two of student colleagues in tow courses to say hello, and make sure to add a signature( see figure 4-5). All these tasks were measured for all three approaches. 


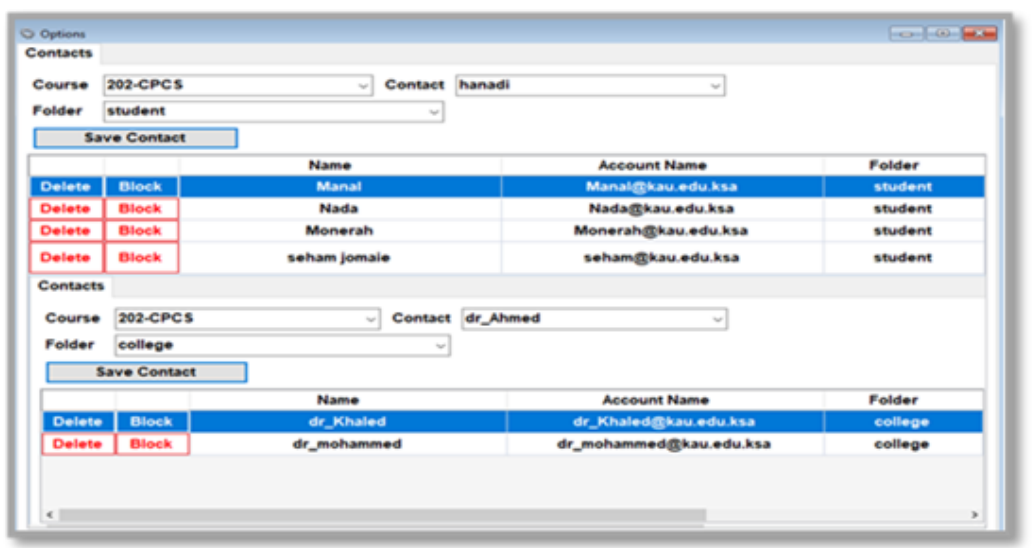

Figure 3. Doctors and students appear in the same course

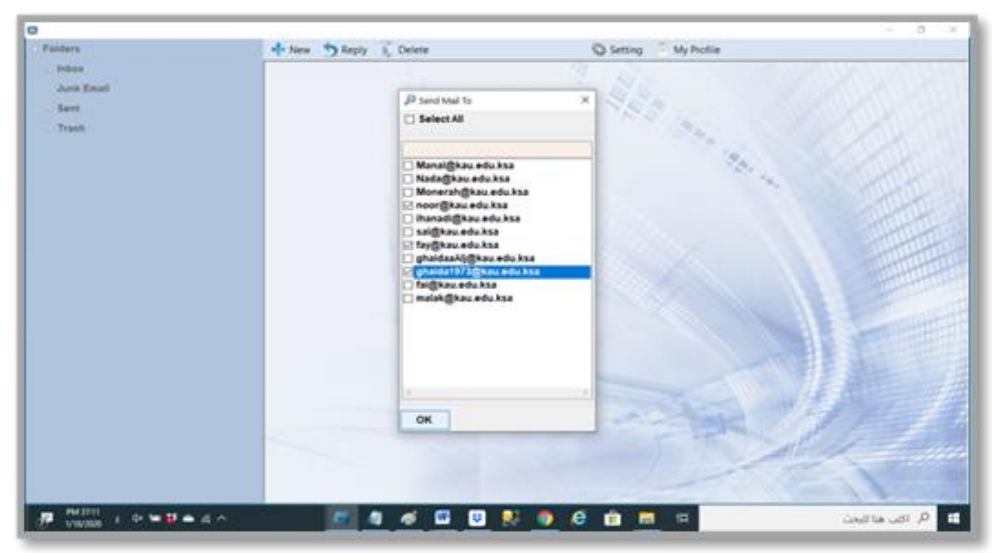

Figure 4. Choosing users address to send messages

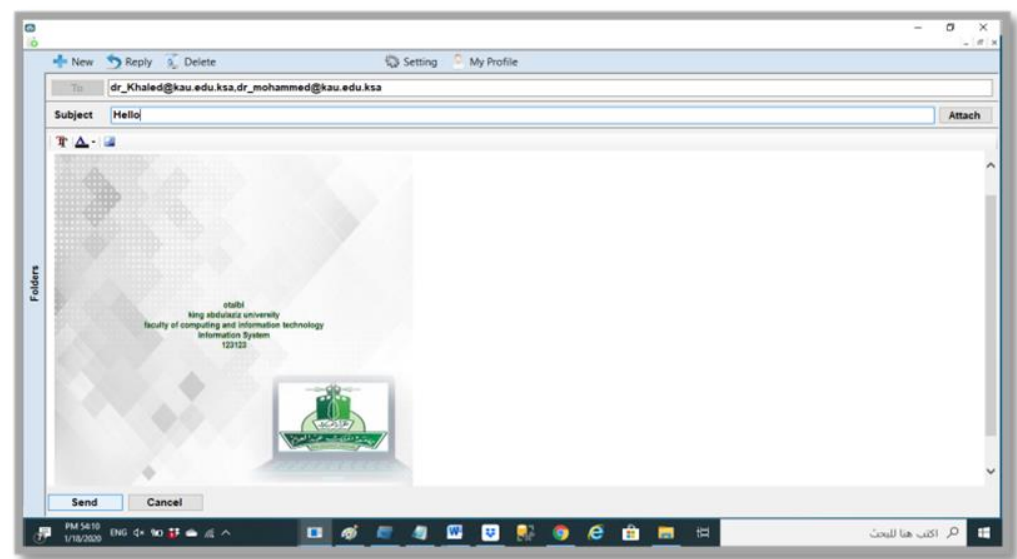

Figure 5. Typing message with signature appearance

\subsection{Independent and Dependent Variables}

Independent variables are the controlled variables during the experiment. They are as follows:

- Tasks: Each subject has a similar set of tasks with the same complexity (easy, medium, and complex): 7 
main tasks and 1 learnable for experiment 1 and 21 main tasks and 3 learnable for experiment 2.

- Interactive metaphors: Interactive metaphors will be assessed by all subjects in all groups.

- Learning effect: All subjects will be assigned randomly to different environments and different task collections to avoid the learning effect.

- Task criterion time: Each task must end within its criterion time. If it is not completed within the criterion time, it would be regarded as an unsuccessfully completed task.

- The amount of training: All subjects will receive the same amount of training and information by recorded training that explains the same amount of information for each group; for example, all groups will see how to arrange all incoming e-mails automatically to different sections (university/college/section). Some groups may get additional training time depending on which approach they test; for instance, respondents who test adaptable condition will take additional guidance on some customization steps.

- Support: Providing a "support" or assist section in the main interface for each approach will ensure the respondents have adequate assistance. Also, it allows answering any questions from subjects any time before and after each task.

The dependent variable is a factor that depends on the independent variable.

We measured and compiled them to test the hypotheses, which are grouped as follows:

- Efficiency

1. Counted errors: the occurrence of errors when performing every task. Only errors in the main tasks will be counted.

2. Counted clicks and visited pages: The number of clicks and pages is needed for each task execution.

3. Task accomplishment time: The total time it takes for a subject to absorb how to execute the task and the time he takes to finish.

- Effectiveness

The total number of respondents who effectively accomplished all tasks: The number of respondents who finished all tasks successfully within the criterion time.

- Satisfaction

General satisfaction: User views are collected during the experiment for each utilized interactive metaphor.

- Customization behavior

1. Customization time: The total time spent by the subject to customize the interface Customization time: The total time spent by the subject to customize the interface

2. Number of clicks required to add the faculty's and colleagues' e-mails, choose the announcements and courses, etc.: The clicks were counted manually.

\subsection{Procedure}

The session duration ranged from 35 to 49 minutes, and the tasks were designed to suit respondents efficiently. The experimental procedure was as follows: (1) An initial questionnaire was used to acquire subjects' demographic information and their experience with computers and customization before the experiment. (2) A 5-minute tutorial about each condition was given to subjects on how to use the experimental system and describe the specialties of each approach. (3) After that, guidance was provided before each set of tasks to give the subjects an opportunity to get used to each approach. Furthermore, subjects were informed that they could ask any question concerning each approach or any experimental procedure. (4) Subjects were asked to evaluate the environments that were being tested at the end of each session. On the other hand, each user's performance was monitored, recorded, and referred to in an assessment form. (5) Once the tasks were completed, respondents were required to finish the questionnaire in order to provide their own views regarding the previous tasks and approaches. In the case with the adaptable approach, the subjects were encouraged to customize the interface within the experiment as a condition to accomplish the tasks. In addition, subjects were supported by instructions on customization and any assistance they needed. When executing the adaptive condition, users were required to register with the system within the experiment, for which they were provided with registration guidance and assistance when needed. Lastly, for the mixed approach, respondents needed to complete two points: first, register with the system as with the adaptive approach, and second, customize the system after reading the experimental scenario. 


\subsection{Trining}

Before starting the experiments, each subject should attend a five-minute training course on their environment as a basic step and provide further clarification as needed.

\subsection{Data Collection}

Both qualitative and quantitative data will be gathered by recording experimental data, discussions, observations, questions, and fillings notes. The benefit of recording the experiments is that any errors made by the users can be documented and every detail of their performance can be evaluated. The subjects will not be told that the experiment will be recorded, so as to protect them from distractions. Qualitative data will be obtained through questionnaires and interviews, where the respondents will be asked to share their views on several aspects of the experiment, such as their satisfaction. The calculations will include accurate calculations of task completion times and the frequency of page visits and clicks. Writing notes and recording observations during the experiment is very helpful for collecting the necessary data and providing a full analysis of all the conditions. All of these measures are grouped and defined as below.

\section{Results}

Usability is necessary to make the navigational process more friendly and effective to meet users' satisfaction. Precisely, it assists the user to reach his goals and maintain his interest in using the website (Roy \& Pattnaik, 2014).

The goal of the study was to measure usability in three main aspects. The first was to evaluate the systems' efficiency regarding each approach by calculating the number of visited pages, clicks, errors, and completed tasks in each approach. Next, the experiments precisely measured effectiveness by calculating the number of respondents who successfully completed all the tasks, in addition to the number of tasks accomplished successfully by all respondents. Finally, respondents' assessments were obtained regarding the ease of use of successfully getting the important messages that mattered to the users, getting faculty email addresses successfully, and organizing incoming mail automatically into multiple sections, as well as general satisfaction.

Efficiency is defined as a measure of the effort required to accomplish a task (Faulkner, 2000) (Frekjmr \& Copenhagen, 2000) (Janicki, Narula, Ziegler, Guénard, \& Economo, 2016). The current study measures efficiency in terms of time required to finish the tasks, the number of pages and clicks, in addition to the total number of errors within the task session. Effectiveness is the capability of achieving goals or completing tasks successfully (Faulkner, 2000) (Frekjmr \& Copenhagen, 2000). It can be calculated as the percentage of respondents who completed all the tasks, or as the percentage of tasks accomplished by all respondents. Each environment is compared separately in terms of effectiveness by extracting a critical time for task accomplishment in each task level. Therefore, if respondents completed a task within the criterion time, that task would be considered as successfully accomplished. Satisfaction is commonly evaluated qualitatively on attitude rating scales by asking every respondent to express their satisfaction with each interaction condition (Frekjmr \& Copenhagen, 2000) (Faulkner, 2000).

\subsection{In Experiment One}

These results are shows after proceeding the experiment by 10 respondents.

\subsubsection{Efficiency}

Figure 7 reveals the mean values of complex, medium, and easy tasks' accomplishment time for both personalized and university email. In the personalized email, users needed only 49 seconds to complete the easy tasks, while in the university email, they spent 207 seconds finishing the tasks. To complete the medium tasks, they needed only 99 seconds in the personalized email, while in the university email, they spent 219 seconds. Moreover, to complete the complex tasks, users needed only 54 seconds in the personalized email, but 297 in the university email.

Furthermore, it can be seen that respondents took longer to accomplish every task at all levels in the university email. Precisely, in medium tasks, respondents took approximately twice as much time in the university email compared with the personalized approach. Moreover, in the personalized email, it took them the same amount of time to execute easy and complex tasks. In the end, the personalized email had faster performance, since it had a useful personalized technique to accomplish all students' tasks without any confusion. 


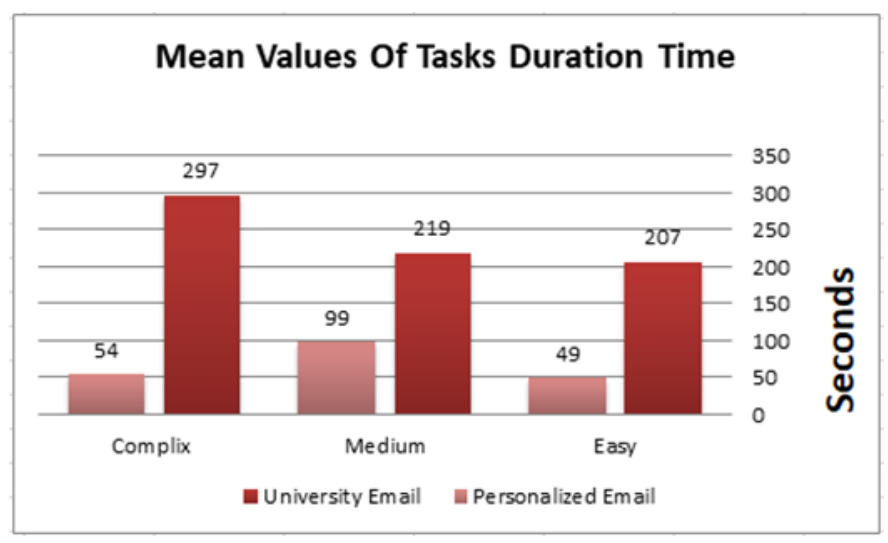

Figure 1. Shows the mean values of the tasks accomplished time

The results in Table 1, reveal that, there is a statistically significant difference between university email and personalized email at the significant level $(0.01)$ for the complex task. Furthermore, the difference is positive to the side of university email, meaning that, respondent who execute complex task in personalized email were faster compared to execute the same tasks with university email.

Table 1. Examining Significant Difference between University and Personalized Email for Complex Tasks

\begin{tabular}{ccccccc}
\hline & $\mathrm{N}$ & Mean & $\mathrm{SD}$ & T-statistics & Df & P-value \\
\cline { 2 - 7 } & 10 & 7.64 & 4.45 & $4.77^{* *}$ & 18 & 0.00 \\
University email & 10 & 0.906 & 0.39 & & \\
\hline
\end{tabular}

**Indicated that the result is significant at the $\mathrm{p}=0.01$ level

The results in Table 2, found that there is a statistically significant difference between university email and personalized email for the easy task, as the significant level is less than the $(0.05)$ i.e. $(\alpha<0.05)$. it was noticeable, executing these tasks took less time compared with university email, which means that the personalized email is better to conduct the tasks successfully.

Table 2. Examining Significant Difference between University and Personalized Email for Easy Tasks

\begin{tabular}{ccccccc}
\hline & $\mathrm{N}$ & Mean & $\mathrm{SD}$ & T-statistics & Df & P-value \\
\hline University email & 10 & 8.07 & 1.57 & \multirow{2}{*}{$11.54^{* *}$} & \multirow{2}{*}{18} & \multirow{2}{*}{0.00} \\
Personalized email & 10 & 1.48 & 0.89 &
\end{tabular}

**Indicated that the result is significant at the $\mathrm{p}=0.01$ level

The results in Table 3, illustrate that there is a statistically significant difference between university email and personalized email at a significant level $(0.01)$ for the medium task. In addition, the results show that the difference is positive to the side of the university email. Which means respondents who execute the medium task in the personalized email were faster compared to execute the same tasks with university email.

Table 3. Examining Significant Difference between University and Personalized Email for Medium Tasks

\begin{tabular}{ccccccc}
\hline & $\mathrm{N}$ & Mean & SD & T-statistics & Df & P-value \\
\hline University email & 10 & 6.78 & 1.33 & $6.477^{* *}$ & 18 & 0.00 \\
Personalized email & 10 & 2.68 & 1.50 & & \\
\hline
\end{tabular}

**Indicated that the result is significant at the $p=0.01$ level

The results in Table 4, reveal that the highest average number of clicks per task (21) and pages visited (4) were in university email, in contrast to personalized email which shows (11) clicks and (2) visited pages per task. 
Table 4. Examining Mean Number of Visited Pages and Clicks

\begin{tabular}{ccc}
\hline Email Type & Number of clicks & Number of visited pages \\
\hline University email & 21 & 4 \\
Personalized email & 11 & 2 \\
\hline
\end{tabular}

\subsubsection{Error Rate}

Respondents made fewer errors in the personalized email than in the university email. The results, as shown in Table 5, indicate that errors occurred in the university email because the students claimed that the interface was confusing and some features were hidden. Surprisingly, some of them didn't know about some features in the university email, such as the signature and the ability to arrange the folders. Furthermore, respondents were lazy and didn't have time to read all emails from their college and university announcements or search for their colleagues and professors' emails, or for different personalized features in the interface.

Table 5. Examining The Frequency of Errors under Personalized and University Email

\begin{tabular}{ccc}
\hline Criteria & Personalized email & University email \\
\hline Incorrect path & 12 & 19 \\
Incorrect category & 6 & 25 \\
Confusing & 16 & 29 \\
Typing error & 6 & 6 \\
Failed task & 2 & 55 \\
Total & 52 & 125 \\
\hline
\end{tabular}

\subsubsection{Effectiveness}

It is apparent from Table 6 that fewer users completed easy, medium, and complex tasks in the university email than in the personalized email. Comparing the two results, as can be seen from the table VI the overall number of users who completed the tasks successfully in the personalized email was 8 out of 10 users, and the overall number of users who completed tasks successfully in the university email was only 1 out of 10 users. Furthermore, on the medium and complex levels, the personalized email had 10 successful users and 40 successfully completed tasks, in contrast to university email, with 0 users and 5 tasks. Moreover, on easy tasks, the personalized email had 8 successful users and 28 successfully completed tasks, compared to the university email, with only 1 user and 12 tasks. Overall, these results indicate that the personalized email was more effective than the existing university email.

Table 6. Examining The Number of Users and Tasks Completed Successfully under Personalized and University Email

\begin{tabular}{cccc}
\hline Tasks Type & $\#$ & Personalized email & University email \\
\hline Complex & Users & 10 out of 10 & 0 out of 10 \\
& Tasks & 20 out of 20 & 1 out of 20 \\
Medium & Users & 10 out of 10 & 0 out of 10 \\
& Tasks & 20 out of 20 & 4 out of 20 \\
Easy & Users & 8 out of 10 & 1 out of 10 \\
& Tasks & 28 out of 30 & 12 out of 30 \\
Overall & Users & 8 from 10 & 1 from 10 \\
& Tasks & 68 from 70 & 17 from 70 \\
\hline
\end{tabular}

\subsubsection{User Satisfaction}

We carried out a questionnaire on experiment 1 after the respondents finished their sessions. This questionnaire was divided into three sections. The first section contained statements with a 6-point Likert rating scale concerning the ease of sending email to professors in both personalized and university email, where 1 indicated extremely easy and 6 not at all easy. Tables 7. and 8. reveal the data acquired along with the percentage of respondents who agreed and disagreed with each statement on the university and personalized email. Moreover, in the second section, the respondents also had to select between 1 (strong agreement) and 6 (strong disagreement) to assess statements on the suitability and presence of new features in the personalized university email. The last section consisted of multiple-choice statements to assess which email was best for college students to use. Based on Table 7, the mean value of respondent replies (statements 1 and 2) concerning ease of 
use was higher for personalized email, at $90 \%$.

Table 7. Examining Respondents' Agreement and Disagreement with Each Statement for the Personalized and University Email (Percentage)

\begin{tabular}{|c|c|c|}
\hline I. Statements & $\begin{array}{l}\text { Extremely } \\
\text { Easy }\end{array}$ & Not At All Easy \\
\hline The Ease of send email to doctors by university email & $40 \%$ & $60 \%$ \\
\hline The Ease of send email to doctors by personalized email & $90 \%$ & $10 \%$ \\
\hline II. Statements & Agree & Disagree \\
\hline $\begin{array}{l}\text { The arranged folders by (university/college/section) in } \\
\text { personalized email are very suitable }\end{array}$ & $80 \%$ & $20 \%$ \\
\hline $\begin{array}{c}\text { It was useful that I can control all received announcements } \\
\text { and messages }\end{array}$ & $90 \%$ & $10 \%$ \\
\hline $\begin{array}{c}\text { The presence of my signature gives a special character to my } \\
\text { messages }\end{array}$ & $90 \%$ & $10 \%$ \\
\hline $\begin{array}{c}\text { Exist faculty and students email and linked with courses was } \\
\text { very efficient }\end{array}$ & $70 \%$ & $30 \%$ \\
\hline
\end{tabular}

Furthermore, the respondents all agreed with adding new features to the personalized university email, as shown in Table 8.

Table 8. Examining Respondents' Views with Each Statement for the Personalized and University Email (Percentage)

\begin{tabular}{ccc}
\hline I. Statements & $\begin{array}{c}\text { Personalized } \\
\text { email }\end{array}$ & $\begin{array}{c}\text { University } \\
\text { email }\end{array}$ \\
\hline Choose the easiest & $100 \%$ & $0 \%$ \\
II. Statements & Yes & No \\
Do you like to have a university email with a personalized system techniques & $90 \%$ & $10 \%$ \\
Was the personalized email suits what do you need & $100 \%$ & $0 \%$ \\
\hline
\end{tabular}

\subsection{In Experiment two}

These results are shows after proceeding the experiment by 30 respondents.

\subsubsection{Efficiency}

Efficiency was measured to determine task accomplishment time for all approaches. Figure 8 illustrates the mean value for how long it took to accomplish complex, medium, and easy tasks. In the adaptive approach, users needed only 34 seconds to complete the easy tasks, and in the adaptable and mixed approaches, they spent 37 seconds finishing the easy tasks. To complete the medium tasks, users needed only 36 seconds in the adaptive approach, while in the adaptable and mixed approaches, they spent 99 and 60 seconds, respectively. Moreover, to complete the complex tasks, users needed only 71 seconds in the adaptive approach, while in the adaptable and mixed approaches, they spent 170 and 88 seconds, respectively.

As can be seen from Figure 8, respondents who executed the adaptable approach spent the longest time on all complexity levels except the easy level, which was equal to the mixed-initiative and close to the adaptive approach. This indicates that there is no observed effect on the easy tasks under the three approaches. Moreover, on complex tasks, respondents took twice as long in the adaptable approach compared with other conditions. Similarly, with the medium level, the adaptable approach took the respondents the longest time. Finally, it can be noted that respondents in the adaptive and mixed approaches were faster at performing all the tasks than those in the adaptive condition. 


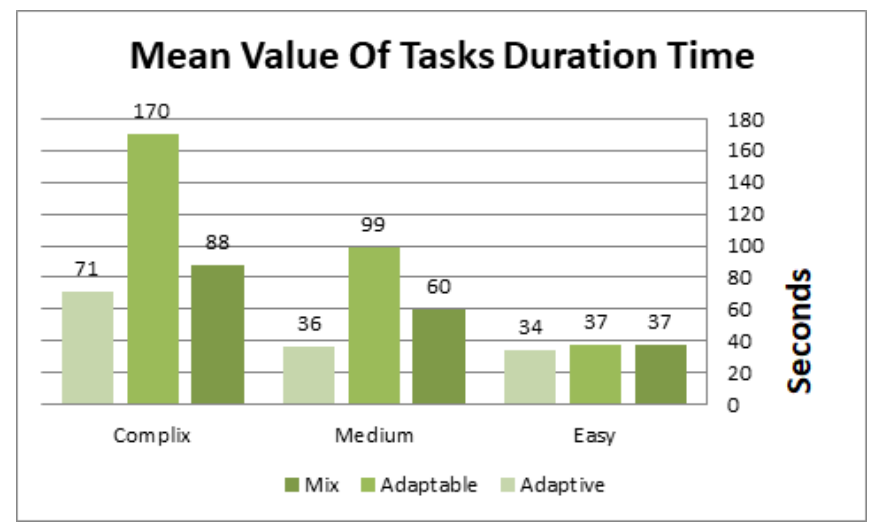

Figure 8. Shows the mean values of the tasks accomplished time

The significant of differences was tested between the three approaches (mix, adaptable, and adaptive). To do that One-Way Analysis of Variance test was run, and the results are demonstrated in Table 9. The F-statistics value is equal to (30.571) which is significant at the (0.01). This result indicated that, there was a significant difference between the tasks related to the approaches (mix, adaptable, and adaptive) at the significant level $(0.01)$.

Table 9. Examining Significant Difference by ANOVA Test between the Three Approaches

\begin{tabular}{cccccc}
\hline & Sum of Squares & df & Mean & \multirow{2}{*}{ Square } & P-value \\
\cline { 2 - 6 } Between Groups & 1099.748 & 2 & 549.874 & & .000 \\
Within Groups & 1564.867 & 87 & 17.987 & $30.571 * *$ & \\
Total & 2664.615 & 89 & & & \\
\hline
\end{tabular}

**Indicated that the result is significant at the $\mathrm{p}=0.01$ level

To examine to which task the differences are significant, multiple comparison analysis was run by using the Least Square Difference (L.S.D) method, and the results presented in Table 10. The difference is significant between the approaches mix, and adaptive, and positive to the side of mix. Also, the result reveal that the difference is significant between adaptable, and both mix, and adaptive, and positive to the side of adaptable. This mean that, the mean value of adaptable is exceeding the mean values of both mix and adaptive.

Table 10. The Results of Multiple Comparison Analysis

\begin{tabular}{ccccc}
\hline Tasks & Mean & \multicolumn{3}{c}{ Mean difference } \\
\cline { 3 - 5 } Mix & 6.83 & - & Mdaptable & Adaptive \\
\cline { 3 - 5 } Adaptable & 12.34 & $5.51^{* *}$ & $-5.51^{* *}$ & $2.92^{* *}$ \\
Adaptive & 3.91 & -2.92 & - & $8.43^{* *}$ \\
\hline
\end{tabular}

The results of the T-test would be run to examine the significant difference between tasks according to the following approaches: Mix - adaptable; Mix - adaptive; Adaptable - adaptive. The results of the T-test are displayed as shown in the following:

Table 11 is quite revealing that, T-test statistics value is equal to (-4.17) which is significant at the (0.01) level. This indicate that there is a significant difference between tasks related to the approaches mix, and adaptable, and the difference is positive to the side of adaptable approaches, as its mean value is reaching (12.34) compared with mean value of mix (6.83). In particular, respondent who execute tasks in mix approach were faster compared to execute the same tasks with adaptable approach. 
Table 11. Examining Significant Difference between Tasks Related to (Mix and Adaptable)

\begin{tabular}{ccccccc}
\hline Approaches & No & Mean & SD & T-statistics & Df & P-value \\
\hline Mix & 30 & 6.83 & 2.43 & $-4.17^{* *}$ & 58 & 0.00 \\
Adaptable & 30 & 12.34 & 6.82 & & \\
\hline
\end{tabular}

**Indicated that the result is significant at the $\mathrm{p}=0.01$ level

As Table 12 shows, the value of T-test statistics is reaching (5.847) which is statistically significant at the (0.01) as the $(\alpha<0.01)$. This indicated that there is a statistically significant difference between the task related to the approaches mix and adaptive. In addition to the difference is positive to the side of the mix condition. Meaning that respondents who execute tasks in an adaptive approach were faster compared to execute the same tasks with mix approach.

Table 12. Examining Significant Difference between Tasks Related to (Mix and Adaptive)

\begin{tabular}{ccccccc}
\hline Approaches & No & Mean & SD & T-statistics & Df & P-value \\
\hline Mix & 30 & 6.83 & 2.43 & $5.847^{* *}$ & 58 & 0.00 \\
Adaptive & 30 & 3.91 & 1.24 & \\
\hline
\end{tabular}

**Indicated that the result is significant at the $\mathrm{p}=0.01$ level

From the data in Table 13, it is apparent that the value of T-test statistics is equal (6.662) which is statistically significant at the $(0.01)$ as the $(\alpha<0.01)$. This indicated that there is a statistically significant difference between the tasks related to the two approaches adaptable and adaptive. Besides, the difference is positive to the side of the adaptable, as the mean value shows. Meaning that respondents who execute tasks in an adaptive approach were faster compared to execute the same tasks with the adaptable approach.

Table 13. Examining Significant Difference between Tasks related to (Adaptable and Adaptive )

\begin{tabular}{ccccccc}
\hline Approaches & No & Mean & SD & T-statistics & Df & P-value \\
\hline Adaptable & 30 & 12.34 & 2.43 & \multirow{2}{*}{$6.662 * *$} & 58 & 0.00 \\
Adaptive & 30 & 3.91 & 6.82 & & \\
\hline
\end{tabular}

**Indicated that the result is significant at the $\mathrm{p}=0.01$ level

Table 14, illustrate the highest average number of visited pages (6) and clicks (27) were in adaptable approach, followed by the mix approach, with (4) pages and (14) clicks, then the adaptive approach with (11) clicks and (3) visited pages since it had a fewest functions.

Table14. Examining Mean Number of Visited Pages and Clicks

\begin{tabular}{ccc}
\hline Email Type & Number of clicks & Number of visited pages \\
\hline Adaptive & 11 & 3 \\
Adaptable & 27 & 6 \\
Mix & 14 & 4 \\
\hline
\end{tabular}

\subsubsection{Error Rate}

The results, as shown in Table 15, indicate that respondents made fewer errors in the adaptive approach. In contrast to adaptable approach, which has the most errors due to many reasons, such as the respondents were bored and confused to do all customized steps like choosing the exact doctor's name or courses especially to accomplish medium and complex tasks. 
Table 15. Demonstrate the Frequency of Errors under the Different Approaches

\begin{tabular}{cccc}
\hline Criteria & Adaptive & Adaptable & Mix \\
\hline Incorrect path & 39 & 49 & 41 \\
Incorrect category & 38 & 47 & 40 \\
Confusing & 41 & 47 & 38 \\
Typing error & 11 & 16 & 15 \\
Failed task & 0 & 25 & 4 \\
Total & 129 & 184 & 138 \\
\hline
\end{tabular}

\subsubsection{Effectiveness}

Table 16 is quite revealing in several ways. Interestingly, all users completed all easy and medium tasks successfully in all three approaches. Furthermore, the results show differences only in complex tasks with all three approaches. Precisely, the highest numbers of successful users and tasks completed successfully were in the adaptive approach, with overall 30 users and 120 tasks. The mixed approach recorded close results, with 26 out of 30 users and 116 out of 120 tasks. If we now turn to the adaptable approach, we observe the lowest averages of 9 out of 30 users and 95 out of 120 tasks. In summary, these results show that users who executed the adaptive condition were more effective than those who executed the other approaches. It is difficult to explain this result, but it might be related to the need for further customization steps to be performed that respondents are unwilling to do.

Table 16. Examining Reveal the Number of Users and Tasks Completed Successfully under the Three Approaches

\begin{tabular}{|c|c|c|c|c|}
\hline Tasks Type & \# & Adaptive & Adaptable & Mix \\
\hline \multirow{2}{*}{ Complex } & Users & 30 out of 30 & 9 out of 30 & 26 out of 30 \\
\hline & Tasks & 120 out of 120 & 95 out of 120 & 116 out of 120 \\
\hline \multirow{2}{*}{ Medium } & Users & 30 out of 30 & 30 out of 30 & 30 out of 30 \\
\hline & Tasks & 30 out of 30 & 30 out of 30 & 30 out of 30 \\
\hline \multirow[b]{2}{*}{ Easy } & Users & 30 out of 30 & 30 out of 30 & 30 out of 30 \\
\hline & Tasks & 60 out of 60 & 60 out of 60 & 60 out of 60 \\
\hline \multirow{2}{*}{ Overall } & Users & 30 out of 30 & 9 out of 30 & 26 out of 30 \\
\hline & Tasks & 210 out of 210 & 185 out of 210 & $206 \mathrm{t}$ of 210 \\
\hline
\end{tabular}

\subsubsection{User Satisfaction}

We carried out a questionnaire for experiment 2 after the respondents finished their sessions. The questionnaire was divided into three sections. In the first section, the respondents had to select between 1 and 6 , where 1 indicated extremely easy and 6 not at all easy, to assess the responses. Tables 17 and 18 reveal the data acquired along with the percentage of respondents who agreed and disagreed with each statement in the three approaches. The statements that were measured were on ease of use, ease of performing the tasks, ease of searching for professors' and colleagues' emails, and ease of organizing email folders. In the second section, the respondents also had to select between 1 (strong agreement) and 6 (strong disagreement) to assess suitability and general satisfaction; some of the statements were negative, concerning confusion, nervousness, and difficulty learning. The multiple-choice section contained statements to measure respondents' views on the quickest, preferred, most helpful approach and which one they most disliked.

The mean value of respondent replies (statements 1-5) concerning ease of use, ease of performing the tasks, ease of searching for professors' and colleagues' emails, and ease of organizing email folders was higher for the new personalized email. Also, the mean value of respondents' replies to positive statements $(6,7,11,12)$ concerning suitability and general satisfaction was higher for the personalized email. In contrast, for the negative statements (8-10), only $43.4 \%$ of respondents felt confused, whereas $22.7 \%$ felt nervous performing the tasks, but $96.7 \%$ did not agree that the platform was difficult to learn. Finally, the overall satisfaction took the high value of $93.3 \%$. 
Table 17. Respondents' Agreement and Disagreement with Each Statement for the Approaches (percentage)

\begin{tabular}{ccc} 
I. Statements & Extremely Easy & Not At All Easy \\
\hline Ease of use & $100 \%$ & $0 \%$ \\
\hline Ease of perform the tasks & $90 \%$ & $10 \%$ \\
\hline Ease of search for doctor & $83.3 \%$ & $16.7 \%$ \\
\hline Ease of search for colleagues & $83 \%$ & $17 \%$ \\
\hline Ease of organize emails folders & $80 \%$ & $20 \%$ \\
\hline II. statements & Agree & Disagree \\
\hline Suitability & $60 \%$ & $40 \%$ \\
\hline Easy to control & $86.7 \%$ & $14.3 \%$ \\
\hline Feel confuse & $43.4 \%$ & $56.6 \%$ \\
\hline Feel nervous & $22.7 \%$ & $77.3 \%$ \\
\hline Difficult to learn & $3.3 \%$ & $96.7 \%$ \\
\hline Convenient to organize news & $90 \%$ & $10 \%$ \\
\hline Satisfaction & $93.3 \%$ & $6.7 \%$ \\
\hline
\end{tabular}

In the last section most respondent was prefer adaptive approach more than other approaches in statements (13-17). Furthermore, in statement (16) the major value 63.3\% of disliked approach was for the adaptable.

Table 18. Examining Respondents' Views with Each Statement for the Three Approaches (Percentage)

\begin{tabular}{cccc}
\hline Statements & \multicolumn{3}{c}{ The approaches } \\
\cline { 2 - 4 } & Adaptive & Adaptable & Mix \\
\hline Choose the easiest & $53.3 \%$ & $0 \%$ & $46.7 \%$ \\
Choose the most efficient & $56.7 \%$ & $6.7 \%$ & $36.7 \%$ \\
Choose the preferred approach & $46.7 \%$ & $10 \%$ & $43.3 \%$ \\
Choose the dislike approach & $30 \%$ & $63.3 \%$ & $6.7 \%$ \\
Choose the helpful in sending emails & $53.3 \%$ & $3.3 \%$ & $43.3 \%$ \\
\hline
\end{tabular}

\section{Discussion}

The first objective of this study was to build a framework to measure the usability of the university email against a personalized approach. The initial experiment was carried out using qualitative and quantitative research designs to resolve conflicting findings. By designing some personalized features that had been assessed from the users' point of view in the previous survey (Alwagadani \& Alomar, 2017), and evaluating them by creating a scenario of regular daily tasks for students in different levels of complexity, we have collected clear results from the two environments. To be specific, we measured efficiency by using metrics such as time to accomplish tasks and frequency of errors, whereas effectiveness was measured by calculating the number of subjects completing all tasks and the number of tasks completed successfully within task criterion times. The statistical results show that there is a statistically significant difference between university email and personalized email. In addition, user satisfaction was measured using 6-point Likert scales. The results obtained from the initial experiment by calculating the time to accomplish tasks show that there was a statistically significant difference between university email and personalized email, with the personalized email performing more efficiently than the university email, and the subjects were faster at executing tasks in the personalized approach. Furthermore, errors were reduced by almost $42 \%$, and the number of clicks and visited pages were lower than in the university email. Furthermore, the overall numbers of successful users and tasks completed successfully in the personalized email were 8 out of 10 users and 68 out of 70 tasks, whereas for the university email, the numbers were only 1 out of 10 users and 17 out of 70 respectively, which mean that the overall number of failing users and failed tasks was greater in the university email than in the personalized email. The subjects were also more satisfied when using the personalized email than when using the current system, as was mentioned above. Overall, the evidence from this study suggests that applying personalized techniques had a strong and positive impact on all respondents.

The second objective of our study illustrated which was the best personalized approach that would be most convenient to apply. For this purpose, we investigated the differences between the three personalized approaches: adaptive, adaptable, and mixed-initiative. The experimental results were obtained from both quantitative and qualitative measures, along with self-reported and observed data. In addition, interviews were conducted with subjects when needed.

The framework in the second experiment was built to evaluate the usability factors - efficiency, effectiveness, 
and user satisfaction - for different personalized approaches. Specifically, the experiment measured efficiency by calculating the frequency of errors, time to accomplish tasks, and number of clicks and visited pages. Furthermore, it measured effectiveness by the number of tasks completed and respondents who completed all the tasks successfully. In addition, it evaluated user satisfaction. The most interesting finding was that overall, the adaptive approach had the most significant results in terms of efficiency and effectiveness and was preferred by almost all users. Unexpectedly, respondents were slower at executing the adaptable approach and had more errors (184) compared with the adaptive (129) and mixed (138) approaches. Also, the adaptive condition was more effective than the other approaches given that there were no failing users or failed tasks in the execution, in contrast to the adaptable condition, where 21 out of 30 failed to complete all the task successfully, and the number of the failed tasks was 25 out of 210 tasks. Meanwhile, the mixed approach was more effective than the adaptable condition, with four failed users and four failed tasks.

Furthermore, respondents were more confused and nervous when dealing with the adaptable condition versus the adaptive approach. For example, during the interviews, they said they were happier when they saw all professors' and colleagues' emails available without their intervention and more confident when sending emails to them, and they were pleased when choosing what messages and announcements appeared in their mailbox. Besides, they claimed that adding courses' or users' emails or arranging emails, in addition to any customization task, made them uncomfortable and wasted their time. And most preferred to deal with the mixed-initiative approach, which gave them the system support and the opportunity to customize whenever they needed. The evidence presented thus far supports the idea that user satisfaction depends on the level of controllability provided to users, along with the effort required from users to do their tasks. Only $10 \%$ of the respondents preferred full control, while the largest group (46.7\%) of respondents preferred less control when dealing with their academic communication, and $43.3 \%$ preferred medium control to let them manage the email interface whenever they needed with system support.

\section{Empirically Derived Guidelines}

By addressing the hypothesis presented at the beginning of this study, we can formalize empirically derived guidelines to enhance students' engagement in using the university's e-mail system. Specifically, this section concerns of the usability guiding principles that can be used by the developer to design a more functional university e-mail. These guidelines are related to the obtained experimental results reported in sections 5 .

In general, students had a positive interaction with the presented personalized features. They were very satisfied to have direct access to the e-mail addresses of the faculty and their colleagues without bothering themselves of searching their e-mails on the university website or asking for them. The e-mail addresses will be accessible once the course is added to the user account. Furthermore, students were satisfied with the control feature to choose what kind of message and announcements they would receive, such as general messages from the university and messages concerning their college and section, and whom to receive messages from, such as faculty members or any e-mail address in their mailbox. Likewise, managing these messages by arranged folders, such as university, college, and section, makes students easily deal with the huge number of messages in their inbox. Moreover, a complex e-mail interface prevents students from utilizing many tasks. They should be visually easy and simple. For instance, students were pleased about being able to personalize their message by adding a personal signature which reflects their personality. This feature already exists in the current university e-mail, but all students in the experiment were not aware of it, along with many other features, such as creating mailbox folders. Besides, the personal signature with the students' information that appears on the university or college logo will give a good impression for any student. Many students want to manage their interface theme to make their interface more personalized and make them feel more comfortable.

The experiments recommend that designers consider the differences between the three personalization approaches, in addition to recognizing what techniques is more convenient and what level of control should be provided. Based on the results obtained from Section 5, supporting Hypotheses 1 and 2 stated in section 3, the adaptive approach was the most preferred and best utilized by students. This means that the control level of customization is a system control to save time and effort for students' requirements since they do not use the e-mail for any entertainment purpose.

\section{Conclusion}

The present study was designed to detect the influence of different personalized conditions on university e-mail usage and to measure the usability of each approach. One of the significant findings that emerged from this study was that personalized e-mail was better than the current university e-mail in terms of efficiency, effectiveness, and user satisfaction. The second major finding was that using adaptive approach in executing the tasks was 
faster than both adaptable and mixed approach. However, the adaptable condition was not found to be as efficient as expected. Some evidence showed that students are lazy and do not have time to customize. Therefore, designing the interface for university students need to be simple and need to contain the most necessary features that serve their needs since the main goal is to facilitate educational users' communications and to exchange mutual services. In general, this is the first study which measures different personalized conditions to enhance the usability of university e-mail usage.

\section{References}

Aeiad, E., \& Meziane, F. (2018). An adaptable and personalised E-learning system applied to computer science Programmes design. Education and Information Technologies, 24(2), 1485-1509. https://doi.org/10.1007/s10639-018-9836-x

Akbulut, Y., \& Cardak, C. S. (2012). Adaptive educational hypermedia accommodating learning styles: A content analysis of publications from 2000 to 2011. Computers \& Education, 58(2), 835-842. https://doi.org/10.1016/j.compedu.2011.10.008

Almasri, A. et al. (2019). Intelligent Tutoring Systems Survey for the Period 2000-2018.

Al-Omar, K., \& Rigas, D. (2009). A user performance evaluation of personalised menus. In 2009 Second International Conference on the Applications of Digital Information and Web Technologies, London (pp. 104-109). https://doi.org/10.1109/ICADIWT.2009.5273847

Al-Shumari, M., \& Bella, G. (2014). Online English vocabulary learning on different systems for non-English speakers. In Proceedings ELMAR-2014, Zadar (pp. 1-4). https://doi.org/10.1109/ELMAR.2014.6923374

Alwagadani, B., \& Alomar, K. (2017). Personalizing University Email toward User Acceptance: An Initial Study. International Journal of Computer Applications, 160(5), 17-23. https://doi.org/10.5120/ijca2017913050

Anabalon, D., Flores, A., Mateos, C., Zunino, A., \& Misra, S. (2017). Controlling complexity of web services interfaces through a metrics-driven approach. In International Conference on Computing Networking and Informatics (ICCNI), Lagos (pp. 1-9). https://doi.org/10.1109/ICCNI.2017.8123807

Betts, K. (2009). Lost in translation: Importance of effective communication in online education. Online Journal of Distance Learning Administration, 12, 1-13.

Bunt, A. (2007). Mixed-Initiative Support for Customizing Graphical User Interfaces. The University Of British Columbia.

Bunt, A., Carenini, G., \& Conati, C. (2007). Adaptive Content Presentation for Web. The Adaptive Web: Methods and Strategies of Web Personalization, Lecture Notes in Computer Science, 4321.

Bunt, A., Conati, C., \& McGrenere, J. (2007). Supporting interface customization using a mixed-initiative approach. In Proceedings of the 12th international conference on Intelligent user interfaces (pp. 92-101). https://doi.org/10.1145/1216295.1216317

Burgos, D., Tattersall, C., \& Koper, R. (2007). How to represent adaptation in e-learning with IMS learning design. Interactive Learning Environments, 15(2), 161-170. https://doi.org/10.1080/10494820701343736

Cecchinato, M. E., Bird, J., \& Cox, A. L. (2000). Personalised email tools: a solution to email overload? in CHI'14 Workshop: Personalised Behaviour Change Technologies, 2014.K. Höök, "Steps to take before intelligent user interfaces become real. Interacting with computers, 12, 409-426. https://doi.org/10.1016/S0953-5438(99)00006-5

Chabay, R. W., \& larkin, J. H. (2020). Computer assisted instruction and intelligent tutoring systems: Shared goals and complementary approaches. Routledge .

Clark, B., \& Matthews, J. (2005). Deciding layers: Adaptive composition of layers in a multi-layer user interface.

Connolly, T. M., \& Stansfield, M. (2006). Enhancing eLearning: Using Computer Games to Teach Requirements Collection and Analysis. USAB.

Cypher, A. (1991). Eager: Programming repetitive tasks by example. https://doi.org/10.1145/108844.108850

Demchuk, A., Lytvyn, V., Vysotska, V., \& Dilai, M. (2019). Methods and means of web content personalization for commercial information products distribution. In International Scientific Conference "Intellectual Systems of Decision Making and Problem of Computational Intelligence". Springer, Cham. https://doi.org/10.1007/978-3-030-26474-1_24 
Faulkner, C. (2000). Usability engineering. Houndmills: Macmillan Press.

Federico, P. A. (2000). Learning styles and student attitudes toward various aspects of network-based instruction. Computers in Human Behavior, 16(4), 359-379. https://doi.org/10.1016/S0747-5632(00)00021-2

Findlater, L., \& McGrenere, J. (2004). A comparison of static, adaptive, and adaptable menus. In Proceedings of the 2004 conference on Human factors in computing systems (pp. 89-96). https://doi.org/10.1145/985692.985704

Findlater, L., \& McGrenere, J. (2010). Beyond performance: Feature awareness in personalized interfaces. International Journal of Human-Computer Studies, 68(3), 121-137. https://doi.org/10.1016/j.ijhcs.2009.10.002

Findlater, L., and McGrenere, J. (2007). Evaluating reduced-functionality interfaces according to feature findability and awareness. LECTURE NOTES IN COMPUTER SCIENCE, 4662, 592. https://doi.org/10.1007/978-3-540-74796-3_59

Frekjmr, E., \& Copenhagen, D. (2000). Measuring Usability: Are Effectiveness, Efficiency, and Satisfaction Really Correlated?

Frias-Martinez, E., Chen, S., \& Liu, X. (2009). Evaluation of a personalized digital library based on cognitive styles: Adaptivity vs. adaptability. International Journal of Information Management, 29(1), 48-56. https://doi.org/10.1016/j.ijinfomgt.2008.01.012

Gajos, K. Z., Czerwinski, M., Tan, D. S., \& Weld, D. S. (2006). Exploring the design space for adaptive graphical user interfaces. In Proceedings of the working conference on Advanced visual interfaces (pp. 201-208). https://doi.org/10.1145/1133265.1133306

Graf, S., \& Kinshuk, K. (2007). Providing adaptive courses in learning management systems with respect to learning styles. In Proceedings of the world conference on e-learning in corporate government healthcare and higher education (e-Learn).

Greenberg, S., \& Witten, I. H. (1985). Adaptive personalized interfaces-A question of viability. Behaviour \& Information Technology, 4, 31-45. https://doi.org/10.1080/01449298508901785

Janicki, J., Narula, N., Ziegler, M., Guénard, B., \& Economo, E. P. (2016). Visualizing and interacting with large-volume biodiversity data using client-server web-mapping applications: The design and implementation of antmaps.org. Ecological Informatics, 32, 185-193.

https://doi.org/10.1016/j.ecoinf.2016.02.006

Ji, H., Yun, Y., Lee, S., Kim, K., \& Lim, H. (2017). An adaptable UI/UX considering user's cognitive and behavior information in distributed environment. Cluster Computing, 21(1), 1045-1058. https://doi.org/10.1007/s10586-017-0999-9

Jorritsma, W., Cnossen, F., \& van Ooijen, P. (2015). Adaptive support for user interface customization: a study in radiology. International Journal of Human-Computer Studies, 77, 1-9.

https://doi.org/10.1016/j.ijhcs.2014.12.008

Jumaa, M., Moussa, S. M., \& Khalifa, M. E. (2017). The main aspects of adaptive educational games for normal and disabled/disordered learners: A comprehensive study. In Eighth International Conference on Intelligent Computing and Information Systems (ICICIS), Cairo, (pp. 348-355). https://doi.org/10.1109/INTELCIS.2017.8260061

Kaur, R., \& Sharma, B. (2018). Comparative Study for Evaluating the Usability of Web Based Applications. In 2018 4th International Conference on Computing Sciences (ICCS), Jalandhar (pp. 94-97). https://doi.org/10.1109/ICCS.2018.00023

Leiva-Chinchilla, P., Vega-Alvarado, J., Calvo-Elizondo, A., \& Francesa-Alfaro, A. (2018). Towards Adaptability: Taking Advantage of Existing Tools in a LMS. In 2018 XIII Latin American Conference on Learning Technologies (LACLO), São Paulo, Brazil (pp. 322-328). https://doi.org/10.1109/LACLO.2018.00063

Lim, H., Hooshyar, D., Ji, H., Lee, S., \& Jo, J. (2018). SmartSenior: Automatic Content Personalization Through Semi-supervised Learning. Wireless Personal Communications, 105(2), 461-473. https://doi.org/10.1007/s11277-018-5947-3

Mackay, W. E. (1990). Patterns of sharing customizable software. https://doi.org/10.1145/99332.99356 
Mackay, W. E. (1991). Triggers and barriers to customizing software. https://doi.org/10.1145/108844.108867

MacLean, A., Carter, K., Lövstrand, L., \& Moran, T. (1990). User-tailorable systems: pressing the issues with buttons. https://doi.org/10.1145/97243.97271

MacLean, A., Carter, K., Lövstrand, L., \& Moran, T. (1990). User-tailorable systems: pressing the issues with buttons. In Proceedings of the SIGCHI conference on Human factors in computing systems (pp. 175-182). https://doi.org/10.1145/97243.97271

Mateos, C., Zunino, A., Misra, S., Anabalon, D., \& Flores, A. (2016). Keeping Web Service interface complexity low using an OO metric-based early approach. In 2016 XLII Latin American Computing Conference (CLEI), Valparaiso (pp. 1-12). https://doi.org/10.1109/CLEI.2016.7833366

McDonald, J. E., Molander, M. E., \& Noel, R. W. (1988). Color-coding categories in menus. https://doi.org/10.1145/57167.57183

McGrenere, J. (2002). The design and evaluation of multiple interfaces: A solution for complex software. The University of Toronto.

McLoughlin, C., \& Lee, M. J. (2008). Future learning landscapes: Transforming pedagogy through social software. Innovate: Journal of Online Education, 4, 1.

Murali, M. V., Vishnu T. G., \& Victor, N. (2019). A Collaborative Filtering based Recommender System for Suggesting New Trends in Any Domain of Research. In 2019 5th International Conference on Advanced Computing \& Communication Systems (ICACCS), Coimbatore, India, (pp. 550-553). https://doi.org/10.1109/ICACCS.2019.8728409

Nigel, F., \& Chen, S. Y. (2000). Individual differences hypermedia navigation and learning: an empirical study. Journal of educational multimedia and hypermedia, 9(4), 281-311.

Page, S. R., Johnsgard, T. J., Albert, U., \& Allen, C. D. (1996). User customization of a word processor. In the proceeding of the SIGCHI Conference on Human Factors in Computing Systems (pp. 340-346). https://doi.org/10.1145/238386.238541

Paterno, F., \& Mancini, C. (1999). Designing web interfaces adaptable to different types of use.

Roy, S., \& Pattnaik, P. K. (2014). Some Popular Usability Evaluation Techniques for Websites. In Proceedings of the International Conference on Frontiers of Intelligent Computing: Theory and Applications (FICTA) 2013 Advances in Intelligent Systems and Computing (pp. 535-543). https://doi.org/10.1007/978-3-319-02931-3_61

Sabourin, J. L., \& Lester, J. C. (2014). Affect and Engagement in Game-BasedLearning Environments. IEEE Transactions on Affective Computing, 5(1), 45-56. https://doi.org/10.1109/T-AFFC.2013.27

Sears, A., \& Shneiderman, B. (1998). Split menus: Effectively using selection frequency to organize menus.

Shneiderman, B. (2003). Promoting universal usability with multi-layer interface design. https://doi.org/10.1145/957205.957206

Shneiderman, B., \& Maes, P. (1997). Direct manipulation vs. interface agents. Interactions, 4, 42-61. https://doi.org/10.1145/267505.267514

Soflano, M., Connolly, T. M., \& Hainey, T. (2015). An application of adaptive games-based learning based on learning style to teach SQL. Computers \& Education, 86, 192-211. https://doi.org/10.1016/j.compedu.2015.03.015

Srivastava, M., \& Goyal, M. (2013). Personalization of Automatic E-mail Response for the University System. In Confluence 2013: The Next Generation Information Technology Summit (4th International Conference). https://doi.org/10.1049/cp.2013.2362

Tsandilas, T. (2005). An Empirical Assessment of Adaptation Techniques. https://doi.org/10.1145/1056808.1057079

Wu, K. L., Aggarwal, C. C., \& Yu, P. S. (2000). Personalization with dynamic profiler.

Wu, L., Yu, P., Wei, J., Xie, Y., \& Kishida, H. (2010). A Method for User-Centered Interface Customization and Development of a Prototype System. In Second World Congress on Software Engineering, Wuhan (pp. 197-200). https://doi.org/10.1109/WCSE.2010.46

Zanker, M., Rook, L., \& Jannach, D. (2019). Measuring the impact of online personalisation: Past, present and 
future. International Journal of Human-Computer Studies, 131, 160-168. https://doi.org/10.1016/j.ijhcs.2019.06.006

Zhao, X. (2019). A Study on E-commerce Recommender System Based on Big Data. In 2019 IEEE 4th International Conference on Cloud Computing and Big Data Analysis (ICCCBDA), Chengdu, China, 2019 (pp. 222-226). https://doi.org/10.1109/ICCCBDA.2019.8725694

\section{Copyrights}

Copyright for this article is retained by the author(s), with first publication rights granted to the journal.

This is an open-access article distributed under the terms and conditions of the Creative Commons Attribution license (http://creativecommons.org/licenses/by/4.0/). 